\title{
Quantitative and Functional Antibody Responses to the 13-Valent Conjugate and/or 23-Valent Purified Polysaccharide Vaccine in Aging HIV-Infected Adults
}

\author{
Jennifer A Ohtola ${ }^{1}$, Jessica L Saul-McBeth ${ }^{1}$, Anita S lyer ${ }^{1}$, David J Leggat ${ }^{1}$, Sadik A Khuder ${ }^{1,4}$, Noor M Khaskhely ${ }^{1}$ and MA Julie \\ Westerink $k^{1,2,3 *}$ \\ ${ }^{1}$ Department of Medicine, University of Toledo College of Medicine \& Life Sciences, Toledo, Ohio \\ ${ }^{2}$ Department of Pathology, University of Toledo College of Medicine \& Life Sciences, Toledo, Ohio \\ ${ }^{3}$ Department of Medical Microbiology and Immunology, University of Toledo College of Medicine \& Life Sciences, Toledo, Ohio \\ ${ }^{4}$ Department of Public Health and Preventative Medicine, University of Toledo College of Medicine \& Life Sciences, Toledo, Ohio
}

\begin{abstract}
Background: The number of aging human immunodeficiency virus-infected $(\mathrm{HIV}+)$ individuals living in the United States has substantially grown over the past two decades. Advanced age and HIV infection both increase susceptibility to Streptococcus pneumoniae infection due to B cell dysfunction. The combined impact of these factors on pneumococcal vaccine responses remains unknown.
\end{abstract}

Methods: We assessed serum immunoglobulin (Ig) G and IgM levels and opsonophagocytic killing assay (OPA) titers to pneumococcal serotypes 14 and $23 \mathrm{~F}$ in HIV+ subjects and HIV-uninfected (HIV-) controls $50-65$ years old. $\mathrm{HIV+}$ individuals with CD4 ${ }^{+} \mathrm{T}$ cells/ $\mu \mathrm{l}(\mathrm{CD} 4)>200$ and $\geq 1$ year of antiretroviral therapy (ART) received either a dose of the 13-valent pneumococcal conjugate vaccine followed by the 23-valent pneumococcal polysaccharide vaccine 8 weeks later (PCV/PPV) as currently recommended $(n=15)$ or a single dose of PPV only $(n=22)$. HIV - controls received PCV/PPV $(n=14)$.

Results: HIV+ PCV/PPV and PPV groups exhibited similar increases in IgG levels and OPA titers for both serotypes after immunization. Postvaccination IgM levels for serotype 23F, but not 14 , were significantly higher in HIV+ PCV/PPV compared to PPV groups. IgG and IgM levels for serotype 14 and OPA titers to serotype 23F were significantly reduced in HIV+ compared to HIV- PCV/PPV groups. Serotype-specific IgG levels correlated with OPA titers for all groups.

Conclusions: Our data suggest that the recommended PCV/PPV regimen may not significantly improve quantitative or functional antibody responses compared to PPV only in aging HIV+ subjects. Continued efforts aimed at improving vaccine responses in this high risk population are warranted.

Keywords: HIV infection; Aging; Human; Antibody; Streptococcus pneumoniae; Pneumococcal conjugate vaccine; Pneumococcal polysaccharide vaccine

\section{Introduction}

Streptococcus pneumoniae infections, including pneumonia and invasive pneumococcal disease (IPD), remain a significant cause of HIV-associated morbidity and mortality despite several clinical advances. Widespread pediatric pneumococcal immunization, due to indirect effects, and use of ART have resulted in substantial reductions in IPD incidence [1,2]. However, disease burden persists in HIV+ individuals despite higher CD4 counts and is 20-40 fold higher than in age-matched uninfected individuals $[1,3]$. Increased rates of recurrence and severe infections are also associated with HIV infection $[4,5]$.

The population of aging HIV+ individuals has rapidly expanded due to the success of ART in reducing mortality combined with the increased rate of new diagnoses in older adults [6-8]. Evidence suggests age influences the course of HIV infection by accelerating the development of comorbidities and decreasing the duration of clinical latency in older patients $[9,10]$. An estimated one-half of HIV+ individuals living in the United States are now $\geq 50$ years old [9]. Advanced age is a significant risk factor for pneumococcal disease in HIV+ and HIV- individuals $[3,11]$. Both aging and HIV infection contribute to B cell dysfunction, resulting in decreased responses to vaccination [9-12].

Recommendations for prevention of bacterial pneumonia in HIV+ adults include use of ART, smoking cessation, and vaccination against influenza and S. pneumoniae [13]. Prior vaccination guidelines for
HIV + adults in the United States recommend a single dose of PPV at diagnosis, followed by revaccination 5 years later, and again after age 65 [14]. However, the effectiveness of PPV in HIV+ adults has been a subject of debate $[15,16]$. Several factors impacting efficacy, including timing of vaccination and degree of immunocompromise, remain ill defined.

In an effort to improve protection against pneumococcal infection, PCV was added to the vaccination recommendations for adults with immunocompromising conditions. For HIV+ pneumococcal vaccinenaive individuals and those vaccinated with a primary dose of PPV $\geq 5$ years prior, the Advisory Committee on Immunization Practices (ACIP) recommends a single dose of PCV followed by a dose of PPV at least 8 weeks later [14]. Immunogenicity studies conducted in HIV+

*Corresponding author: MAJ Westerink, Medical University of South Carolina, 135 Rutledge Avenue Suite 1209, PO Box MSC752, Charleston, SC 29425, USA, Tel: 843-792-4541; Fax: 843-792-6680; E-mail: westerin@musc.edu

Received February 10, 2016; Accepted March 03, 2016; Published March 14 2016

Citation: Ohtola JA, Saul-McBeth JL, lyer AS, Leggat DJ, Khuder SA, et al. (2016) Quantitative and Functional Antibody Responses to the 13-Valent Conjugate and/ or 23-Valent Purified Polysaccharide Vaccine in Aging HIV-Infected Adults. J AIDS Clin Res 7: 556. doi:10.4172/2155-6113.1000556

Copyright: ( 2016 Ohtola JA, et al. This is an open-access article distributed unde the terms of the Creative Commons Attribution License, which permits unrestricted use, distribution, and reproduction in any medium, provided the original author and source are credited. 
Citation: Ohtola JA, Saul-McBeth JL, Iyer AS, Leggat DJ, Khuder SA, et al. (2016) Quantitative and Functional Antibody Responses to the 13-Valent Conjugate and/or 23-Valent Purified Polysaccharide Vaccine in Aging HIV-Infected Adults. J AIDS Clin Res 7: 556. doi:10.4172/21556113.1000556

Page 2 of 7

adults have thus far, however, yielded inconsistent results regarding the superiority of PCV alone or in combination with PPV over the prior recommendation [17-23]. Thus, the potential value of $\mathrm{PCV}$ in the HIV+ population remains to be established.

The combined impact of immunosenescence and HIV infection on responses to pneumococcal vaccines may be an important consideration in the clinical management of older HIV+ adults. The goal of the current study was to compare quantitative and functional antibody responses generated from the recommended PCV/PPV regimen to a single dose of PPV in HIV+ adults 50-65 years old.

\section{Methods}

\section{Design and study population}

Volunteers 50-65 years old were recruited between April 2012 and January 2015 at the University of Toledo Medical Center. Written, informed consent was obtained from all subjects. The study was monitored and approved by the Institutional Review Board at the University of Toledo. Exclusion criteria included: active infection (except HIV), PPV <5 years prior, pregnancy, immunosuppressive medications, and history of cancer, autoimmune disease, bleeding disorders, immunoglobulin therapy, organ transplantation, splenectomy, and end stage renal or liver disease. Volunteers were questioned about any prior hospitalizations consistent with pneumococcal infection. Eligibility criteria for HIV+ participants were further defined as current CD4 $>200$, HIV viral load $\leq 400$ copies/ml, and ART for $\geq 1$ year. Adherence to ART was confirmed by patient's self-report and review of medical records. HIVindividuals received a single dose of PCV (Prevnar 13; Wyeth Pharmaceuticals, Inc.) followed by one dose of PPV (Pneumovax $23^{\circ}$; Merck \& Co., Inc.) 8 weeks later (PCV/PPV, n=14). HIV+ individuals received either PCV/PPV $(n=15)$ or a single dose of PPV $(n=22)$. All participants who received PCV/PPV were followed up at 2 months (8 weeks; PPV immunization) and 3 months after study enrollment. Participants who received PPV were followed up at 1 month after enrollment.

\section{Laboratory}

Blood samples were collected at each study visit. Serum samples were used to measure capsular pneumococcal polysaccharide-specific antibody responses pre- and 1 month postvaccination with PPV (post-PPV) to serotypes 14 and $23 \mathrm{~F}$ (included in PCV and PPV). These serotypes were selected due to their historically high prevalence in the HIV+ population and inclusion in our previous studies [24,25]. Serotype-specific IgG and IgM serum levels were detected by enzyme-linked immunosorbent assay (ELISA) as previously described using 89SF or 007SP as standards. Opsonophagocytic killing assay (OPA) was performed as previously described $[26,27]$ to determine functional antibody responses. Data were analyzed using the Opsotiterl software program (University of Alabama at Birmingham). OPA titers were defined as the reciprocal of the serum dilution that killed $50 \%$ of target bacteria (compared to serum-free control) during 45 minutes of incubation at $37^{\circ} \mathrm{C}$.

\section{Statistical Analysis}

Participant characteristics were represented as mean (range) for numerical values and number (percentage) for categorical values. Serotypespecific serum $\operatorname{IgG}$ and IgM levels $(\mu \mathrm{g} / \mathrm{ml})$ and OPA titers were reported as geometric mean concentrations or titers ( $95 \%$ confidence interval), respectively. Responders to vaccination were determined as previously defined $[17,18,20]$. Serotype-specific IgG and IgM responses were defined as a $\geq 2$-fold increase from baseline and post-PPV levels of $\geq 1 \mu \mathrm{g} / \mathrm{ml}$. A positive OPA response was defined as a $\geq 4$-fold increase from baseline post-PPV. IgG and IgM levels and OPA titers were log-transformed to approximate normal distribution prior to statistical analysis. Pre- to 1 month post-PPV comparisons between groups were calculated by analysis of covariance (ANCOVA) with Bonferroni correction to adjust for differences in baseline levels. Post-PPV antibody responses were compared using analysis of variance (ANOVA) with Dunnett's post-hoc test, with HIV+ PCV/PPV designated as the control group. The number of responders from each group were compared using the Fisher's exact test. Correlations were determined by Pearson's correlation coefficient. All statistical analyses were performed using the SAS software package (version 9.3; SAS Institute). $P$ values $<0.05$ were considered significant.

\begin{tabular}{|c|c|c|c|}
\hline \multirow[b]{2}{*}{ Characteristic } & \multicolumn{2}{|c|}{ HIV-infected } & \multirow{2}{*}{$\begin{array}{l}\text { HIV-uninfected } \\
\text { PCV/PPV } \\
(\mathrm{N}=14)\end{array}$} \\
\hline & $\begin{array}{c}\mathrm{PPV} \\
(\mathrm{N}=22)\end{array}$ & $\begin{array}{c}\mathrm{PCV} / \mathrm{PPV} \\
(\mathrm{N}=15)\end{array}$ & \\
\hline \multicolumn{4}{|l|}{ Demographic } \\
\hline Mean age (range) & $55.2(50-64)$ & $54.8(49-63)$ & $55.6(50-64)$ \\
\hline Male (\%) & $20(91)$ & $12(80)$ & $6(43)$ \\
\hline \multicolumn{4}{|l|}{ Race (\%) } \\
\hline $\begin{array}{l}\text { Black } \\
\text { White } \\
\text { Other }\end{array}$ & $\begin{array}{l}7(32) \\
15(68) \\
0(0.0)\end{array}$ & $\begin{array}{l}8(53) \\
5(33) \\
2(13)\end{array}$ & $\begin{array}{c}1(7) \\
12(86) \\
1(7)\end{array}$ \\
\hline \multicolumn{4}{|l|}{ Clinical History } \\
\hline Prior PPV $\geq 5$ years (\%) & $18(82)$ & $13(87)$ & $1(7)$ \\
\hline Receiving $A R T \geq 1$ year (\%) & $22(100)$ & $15(100)$ & N/A \\
\hline \multicolumn{4}{|c|}{ Nadir $\mathrm{CD} 4^{+} \mathrm{T}$ cell count $($ cells/ $/ \mathrm{ll})$} \\
\hline $\begin{array}{l}>200(\%) \\
\leq 200(\%)\end{array}$ & $\begin{array}{l}10(46) \\
12(55)\end{array}$ & $\begin{array}{c}5(33) \\
10(67)\end{array}$ & $\begin{array}{l}\text { N/A } \\
\text { N/A }\end{array}$ \\
\hline \multicolumn{4}{|l|}{ Laboratory Data at Enrollment } \\
\hline \multicolumn{4}{|l|}{ HIV viral load (copies/ml) } \\
\hline$\leq 400(\%)$ & $22(100)$ & $15(100)$ & N/A \\
\hline \multicolumn{4}{|l|}{$\mathrm{CD}^{+} \mathrm{T}$ cell count (cells/ $\left.\mu \mathrm{l}\right)$} \\
\hline Mean (range) & $652(230-1599)$ & $717(331-1298)$ & N/A \\
\hline
\end{tabular}

PCV/PPV groups received PCV followed by PPV 8 weeks later. Data are no. (\%) of subjects, unless otherwise noted.

Abbreviations: HIV: Human Immunodeficiency Virus; PPV 23: Valent Pneumococcal Polysaccharide Vaccine; PCV 13: Valent Pneumococcal Conjugate Vaccine.

Table 1: Baseline Characteristics of Study Participants. 
Citation: Ohtola JA, Saul-McBeth JL, Iyer AS, Leggat DJ, Khuder SA, et al. (2016) Quantitative and Functional Antibody Responses to the 13-Valent Conjugate and/or 23-Valent Purified Polysaccharide Vaccine in Aging HIV-Infected Adults. J AIDS Clin Res 7: 556. doi:10.4172/21556113.1000556

Page 3 of 7

\section{Results}

\section{Subjects}

Baseline characteristics of the 51 participants (37 HIV+ and 14 HIV-) included in this study are reported in Table 1. CD4 counts at enrollment were similar between HIV+ groups. Other clinical characteristics, including nadir CD4 counts and HIV viral load, did not differ between HIV+ groups. All HIV+ subjects were adherent to ART $\geq 1$ year. Differences in the distributions of sex and race in the HIVgroup compared to the HIV+ groups were noted. A larger proportion of HIV+ participants had been immunized with PPV $\geq 5$ years prior $(84 \%)$ compared to HIV- (7\%).

\section{Serum antibody levels to serotypes 14 and $23 \mathrm{~F}$}

Pre- to 1 month post-PPV IgG levels were significantly higher in all groups for both serotypes $(P<0.009$; Figure 1$)$. For IgM, significant increases pre- to post-PPV were observed only for serotype 14 in the $\mathrm{HIV}+\mathrm{PPV}$ group and for serotype $23 \mathrm{~F}$ in the HIV+ PCV/PPV group $(P<0.05)$. IgM levels significantly increased pre- to post-PPV in the HIV- PCV/PPV group for both serotypes $(P<0.001)$.

Post-PPV antibody levels were compared between HIV+ PPV and PCV/PPV groups and between HIV+ and HIV- PCV/PPV groups. In $\mathrm{HIV}+\mathrm{PPV}$ and PCV/PPV groups, post-PPV IgG levels for both serotypes were similar. Post-PPV IgM levels for serotype 23F, but not 14 , were significantly lower in the HIV+ PPV compared to HIV+ PCV/PPV groups $(P<0.05)$. Post-PPV IgG and IgM levels were significantly reduced for serotype 14 only in HIV+ compared to HIV- PCV/PPV groups $(P<0.05)$. The number of subjects that had positive IgG or IgM responses (defined as

\begin{tabular}{|c|c|c|c|}
\hline \multicolumn{1}{|c|}{} & $\begin{array}{c}\text { HIV-infected } \\
\text { PPV }\end{array}$ & $\begin{array}{c}\text { HIV-infected } \\
\text { PCV/PPV }\end{array}$ & $\begin{array}{c}\text { HIV-uninfected } \\
\text { PCV/PPV }\end{array}$ \\
\hline IgG & & & \\
\hline 1 & $10(45.5)$ & $4(26.7)$ & $2(14.3)$ \\
\hline 2 & $6(27.3)$ & $5(33.3)$ & $2(14.3)$ \\
\hline IgM & $6(27.3)$ & $6(40.0)$ & $10(71.4)$ \\
\hline 0 & & & \\
\hline 1 & $20(90.9)$ & $12(80.0)$ & $8(57.1)$ \\
\hline 2 & $2(9.1)$ & $3(20.0)$ & $5(35.7)$ \\
\hline OPA Titer & $0(0)$ & $0(0)$ & $1(7.1)$ \\
\hline 0 & & & $0(0)$ \\
\hline 1 & $2(9.1)$ & $1(6.7)$ & $1(7.1)$ \\
\hline 2 & $17(77.3)$ & $10(66.7)$ & $13(92.9)$ \\
\hline
\end{tabular}

PCV/PPV groups received PCV followed by PPV 8 weeks later. Responders were defined as $\geq 2$-fold increase and $\geq 1 \mu \mathrm{g} / \mathrm{ml}$ in IgG (top panel) and IgM (middle panel) levels or $\geq 4$-fold increase in OPA titers (bottom panel) 1 month postvaccination with PPV. $P \geq 0.05$ for all comparisons between groups. Abbreviations: IgG Immunoglobulin G; IgM: Immunoglobulin M; OPA: Opsonophagocytic Killing Assay; HIV: Human Immunodeficiency Virus; PPV 23: Valent Pneumococcal Polysaccharide Vaccine; PCV: 13-Valent Pneumococcal Conjugate Vaccine.

Table 2: Number (percentage) of responders to the indicated number of serotypes after vaccination.
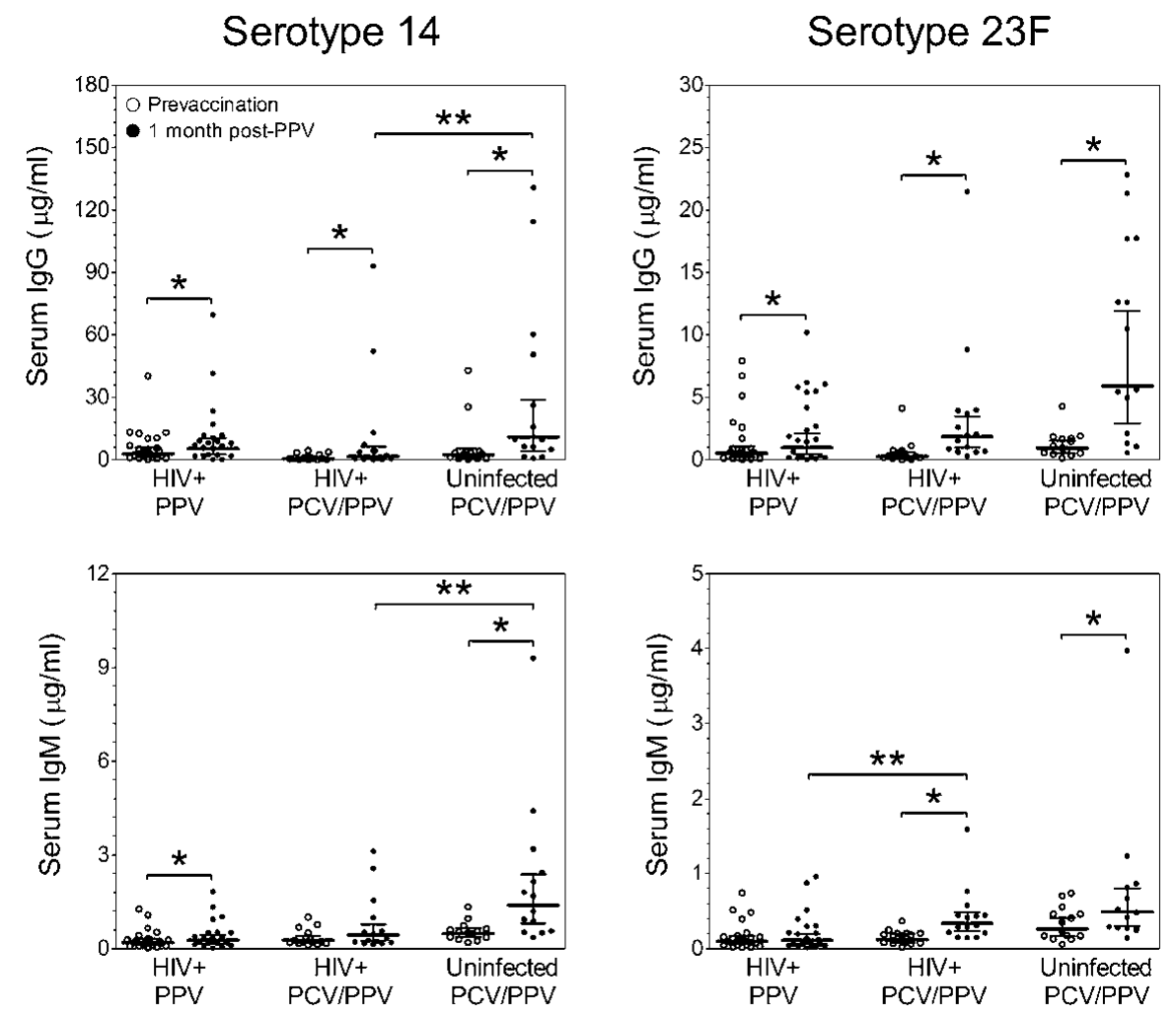

${ }^{\star} P<0.05$ versus prevaccination antibody level. ${ }^{*} P<0.05$ versus post-PPV HIV-infected PCV/PPV antibody level.

Abbreviations: IgG: Immunoglobulin G; IgM: Immunoglobulin M; HIV: Human Immunodeficiency Virus; PPV, 23: Valent Pneumococcal Polysaccharide Vaccine; PCV, 13: Valent Pneumococcal Conjugate Vaccine.

Figure 1: Serotype-specific serum IgG (top panels) and IgM (bottom panels) antibody levels were measured in aging HIV-infected and HIV-uninfected adults. Scatter dot plots include geometric mean concentrations (horizontal black line) and $95 \%$ confidence intervals (error bars). 
Citation: Ohtola JA, Saul-McBeth JL, Iyer AS, Leggat DJ, Khuder SA, et al. (2016) Quantitative and Functional Antibody Responses to the 13-Valent Conjugate and/or 23-Valent Purified Polysaccharide Vaccine in Aging HIV-Infected Adults. J AIDS Clin Res 7: 556. doi:10.4172/21556113.1000556

Page 4 of 7

$\geq 2$-fold increase and post-PPV levels $\geq 1 \mu \mathrm{g} / \mathrm{ml}$ ) were similar for all groups, although more frequent in HIV- individuals (Table 2). Positive responses were lower for IgM than IgG for both serotypes.

\section{Serum OPA titers to serotypes 14 and $23 F$}

Significant increases in serotype-specific OPA titers from pre- to 1 month post-PPV occurred in all groups $(P<0.0001$, Figure 2). Post-PPV OPA titers were compared between HIV+ PPV and PCV/PPV groups and between HIV+ and HIV- PCV/PPV groups. Post-PPV OPA titers were similar between HIV+ PPV and PCV/PPV groups. In HIV+ PCV/ PPV compared to HIV- PCV/PPV groups, post-PPV OPA titers were significantly reduced for serotype $23 \mathrm{~F}$ only $(P<0.05)$. Positive OPA responses (defined as $\geq 4$-fold increase) were similar between all study groups (Table 2).

\section{Correlations between post-PPV antibody levels and OPA titers}

For all groups, there were significant correlations observed between post-PPV serotype-specific IgG levels and OPA titers (Figure 3). There was a strong correlation for serotype $23 \mathrm{~F}$ in the HIV+ PPV group. In the HIV+ PCV/PPV group, there was a strong correlation for serotype 14. Moderate to strong correlations for both serotypes 14 and 23F were observed in the HIV- PCV/PPV group. There were no significant correlations between post-PPV serotype-specific IgM levels and OPA titers in any of the study groups.

\section{Discussion}

The current study is the first to our knowledge to assess the impact of a combined PCV/PPV regimen in older HIV+ individuals. Our findings suggest that this approach may offer no clear improvement in serum antibody titers or opsonophagocytic activity compared to a single dose of PPV. Immunization of HIV+ subjects with either PPV or PCV/PPV resulted in significant but similar increases in serotypespecific IgG levels and OPA titers compared to baseline levels. However, the magnitude of antibody responses in the HIV+ PCV/PPV group was diminished compared to those observed in age-matched HIV- PCV/ PPV controls.

HIV disease progression results in extensive defects in humoral immunity. Loss of memory B cell subsets is associated with impaired antibody responses to pneumococcal vaccination that are incompletely restored by ART [28-30]. We have previously shown that both ARTtreated and -untreated HIV+ adults exhibit reduced serotype-specific IgM memory B cells after PPV compared to HIV- individuals [26,27]. In addition, a substantial proportion of patients on ART do not achieve normalization of CD4 counts [31]. These data, in combination with the current study, suggest that despite higher CD4 counts and use of ART, persistent cellular defects likely contribute to diminished antibody responses after vaccination in our HIV+ subjects.

Independent of HIV infection, increased age is associated with reduced responses to pneumococcal vaccination [11]. Impairment of functional responses in older adults may result from reduced serotypespecific IgM levels post-PPV $[11,24,32]$. We have demonstrated that serotype-specific IgM memory B cells are also reduced in elderly individuals post-PPV compared to younger volunteers [24]. A strength of our study is the use of age-matched uninfected controls. Minimal increases in the frequency of serotype-specific IgM responses were observed for all groups. Therefore, although HIV- participants had higher IgG levels and OPA titers, immunosenescence likely impacted humoral responses in all of our subjects. We investigated peripheral B cell subpopulations responding to PCV and/or PPV in these individuals to further delineate the impact of aging and HIV infection on vaccine responses in a separate study [33].

The finding that PPV and PCV/PPV elicited similar responses is consistent with some $[19,22]$, but not all $[18,20]$, previous studies in HIV+ individuals. Increased immunogenicity with PCV observed in other studies may be of limited duration, however, as the number of responders decline as early as 6 months postvaccination $[17,20]$. The transient nature of antibody responses has also been a longstanding issue with PPV in HIV+ individuals [15]. Potential differences in duration of antibody responses in our study subjects are currently being assessed.

Immunological hyporesponsiveness to repeated vaccination is a potential concern [34]. The majority of our HIV+ participants were previously vaccinated with $\mathrm{PPV}$, in contrast to our HIV- subjects. Although the number of individuals analyzed was limited, we found no differences in antibody responses between HIV+ participants who were vaccine naive and those vaccinated with $P P V \geq 5$ years prior, regardless of whether they received PPV or PCV/PPV. This finding is consistent

\section{Serotype 14}

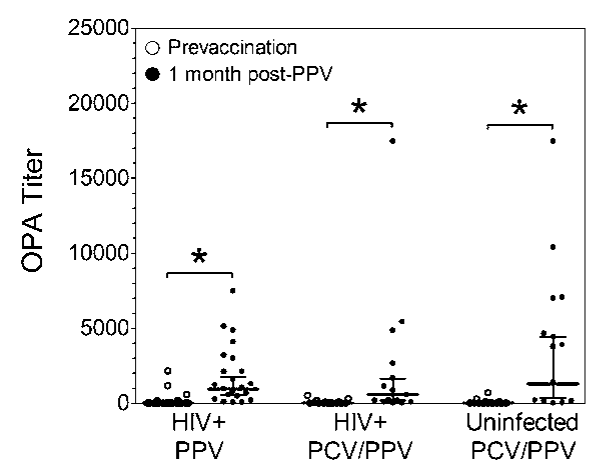

Serotype $23 \mathrm{~F}$

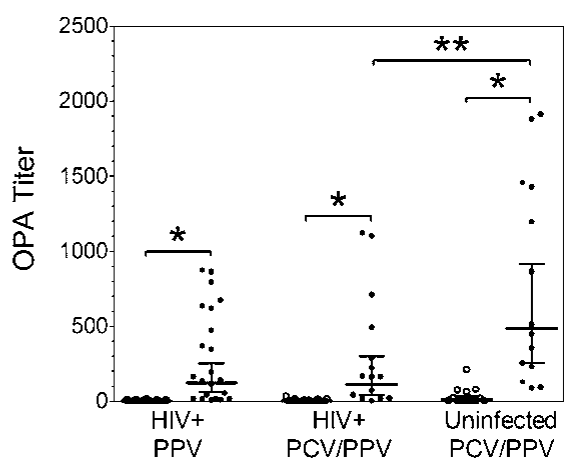

${ }^{*} P<0.05$ versus prevaccination titer, ${ }^{* *} P<0.05$ versus post-PPV HIV-infected PCV/PPV titer

Abbreviations: OPA: Opsonophagocytic Killing Assay; HIV: Human Immunodeficiency Virus; PPV, 23: Valent Pneumococcal Polysaccharide Vaccine; PCV, 13: Valent Pneumococcal Conjugate Vaccine.

Figure 2: Serotype-specific serum OPA titers were determined in aging HIV-infected and HIV-uninfected adults. Scatter dot plots include geometric mean titers (horizontal black line) and 95\% confidence intervals (error bars). 
Citation: Ohtola JA, Saul-McBeth JL, Iyer AS, Leggat DJ, Khuder SA, et al. (2016) Quantitative and Functional Antibody Responses to the 13-Valent Conjugate and/or 23-Valent Purified Polysaccharide Vaccine in Aging HIV-Infected Adults. J AIDS Clin Res 7: 556. doi:10.4172/21556113.1000556

Page 5 of 7

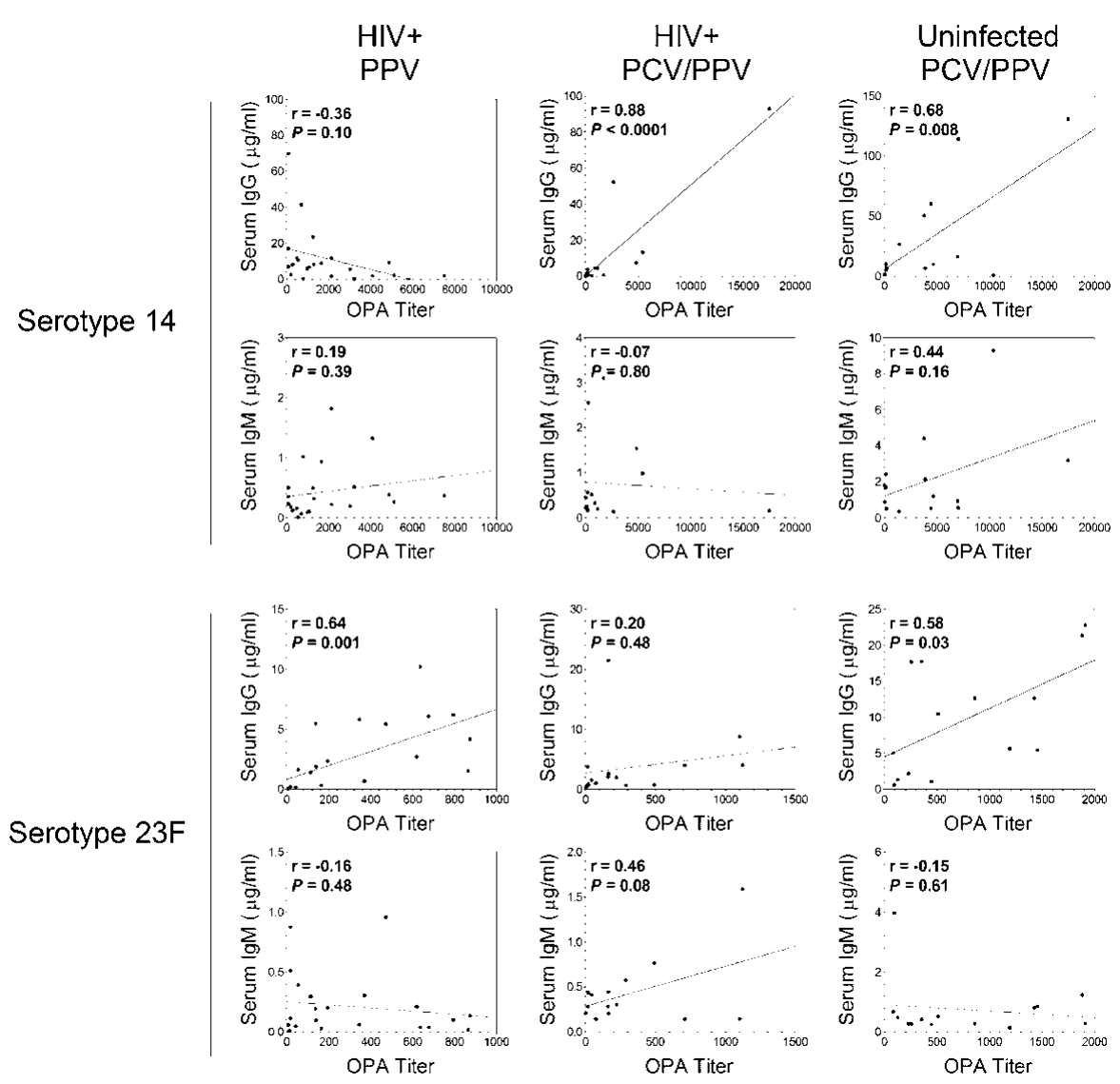

Abbreviations: IgG: Immunoglobulin G; IgM: Immunoglobulin M; HIV: Human immunodeficiency virus; OPA: Opsonophagocytic Killing Assay; PPV, 23: Valent Pneumococcal Polysaccharide Vaccine; PCV, 13: Valent Pneumococcal Conjugate Vaccine.

Figure 3: Correlations between post-PPV antibody levels and OPA titers in aging HIV-infected and HIV-uninfected adults.

with other revaccination studies $[17,21,26,35]$. The diminished antibody responses in our HIV+ subjects are therefore unlikely due to prior vaccination.

Similar to previous findings, we observed increases in antibody responses after PCV that were not significantly enhanced by the subsequent dose of PPV (data not shown) [18,19]. As an alternative to single doses or combinations of PCV and PPV, several studies have evaluated antibody responses in HIV+ individuals given consecutive doses of PCV $[18,21,23,35]$. Additional doses of PCV after an initial dose appear to have limited impact on antibody responses. Several issues regarding this approach remain, including optimal dosing intervals, number of PCV boosters, and limited serotype coverage of PCV compared to PPV. Increased incidence of non-PCV serotypes continues to be a concern, particularly for high-risk populations [2,36]. Thus, it remains unclear how PCV may be utilized in aging HIV+ individuals to improve antibody responses and protection against disease.

Vaccine responses were assessed in the current study using established immunological parameters. We found significant correlations between serotype-specific IgG concentrations and OPA titers post-PPV; however, we also observed OPA responses in individuals lacking a positive IgG response. Discrepancies between these assays have been reported in several adult populations including the elderly and immunocompromised. The OPA assay is generally regarded as a better measure of protection compared to antibody concentrations as it mimics the host phagocytic response [37]. Serological criteria for evaluation of pneumococcal vaccines in infants have been established, but correlates of protective pneumococcal immunity in adult populations are lacking [38]. Thus, it is possible that PCV/PPV elicited better protection in HIV+ subjects compared to PPV even though conventional assays did not indicate it, emphasizing that defined criteria to predict protection are urgently needed in adults.

Pneumococcal disease is preceded by asymptomatic nasopharyngeal carriage. PPV does not appear to affect pneumococcal colonization [39]. Several studies indicate that conjugate vaccines reduce acquisition of vaccine-type carriage in vaccinated children, resulting in decreased transmission of vaccine-type serotypes to adults [39-41]. PCV may also directly reduce nasopharyngeal colonization in adult populations. Immunological parameters currently utilized in immunogenicity studies exclude any potential impact of PCV on mucosal defenses that could contribute to colonization and protective immunity. Increases in serotype-specific IgG or IgA concentrations have been detected in the lung fluid of HIV+ subjects and saliva of immunocompetent adults following conjugate vaccination [42,43]. Presently, mucosal antibody levels and nasopharyngeal colonization are not routinely measured in immunogenicity studies or efficacy trials, but should be considered as possible measures of protection in addition to ELISA and OPA assays.

Clinical trials evaluating the efficacy of PPV in HIV+ adults have 
Citation: Ohtola JA, Saul-McBeth JL, lyer AS, Leggat DJ, Khuder SA, et al. (2016) Quantitative and Functional Antibody Responses to the 13-Valent Conjugate and/or 23-Valent Purified Polysaccharide Vaccine in Aging HIV-Infected Adults. J AIDS Clin Res 7: 556. doi:10.4172/21556113.1000556

failed to demonstrate a clear reduction in pneumococcal disease $[15,16]$. While one study demonstrated vaccine efficacy of $49 \%$ against IPD [44], another trial in Uganda reported possible detrimental effects [45]. The only trial among HIV+ adults examining the conjugate vaccine to date found $74 \%$ efficacy against recurrent vaccine-type IPD; however, protection was greatest within the first year only [46]. A 9-valent conjugate vaccine administered to HIV+ children also reduced vaccine-type IPD, but had no significant impact on pneumonia [47]. In elderly individuals, PPV reduced the risk of IPD with an estimated 55\% efficacy, but its effectiveness in preventing nonbacteremic pneumonia is controversial $[48,49]$. Recently, a large randomized trial conducted in the Netherlands examining the impact of PCV in older adults reported a vaccine efficacy of $75 \%$ for vaccine-type IPD and $45 \%$ for vaccine-type nonbacteremic community-acquired pneumonia [50]. However, the study did not include comparison with PPV. Large scale efficacy trials evaluating PCV versus PPV in aging HIV+ individuals are unlikely. Lack of clear, direct clinical evidence that PCV or PPV provides protection against all vaccine-type pneumococcal disease in older and HIV+ adults further emphasizes the need for studies investigating immunological mechanisms responsible for increased risk and development of alternative vaccination approaches.

We recognize that our study has several limitations. Our sample size was small, limiting the power of the current study. In a separate study, we observed that PCV did not enhance cellular responses to vaccination with PPV in these subjects [33], supporting the findings of noninferiority between vaccination regimens in the current manuscript at the antibody level. Our study evaluated only 2 serotypes, and thus it is unknown what impact an initial dose of PCV may have on other serotypes. We selected serotypes 14 and $23 \mathrm{~F}$ based on their differences in immunogenicity and inclusion in both PCV and PPV. In addition, we did not measure serum IgA concentrations, as IgA levels in respiratory mucosal tissues, and not serum, are likely to confer protection. It has been shown that serum IgA levels do not correlate with salivary IgA levels in adults immunized with conjugate vaccine [43].

In conclusion, we determined that PCV/PPV may not demonstrate a clear immunological advantage compared to PPV alone in older $\mathrm{HIV}+$ individuals, and antibody responses to PCV/PPV were reduced compared to HIV- PCV/PPV controls. Development of more effective vaccination strategies in the aging HIV+ population may be very challenging due to the presence of multiple comorbidities that increase risk of pneumococcal infection. Continued efforts aimed at defining correlates of vaccine protection in adults and immunological mechanisms that reduce vaccine responses are critical.

\section{Acknowledgements}

We thank all study volunteers for their participation and the Division of Infectious Diseases physicians and staff at the University of Toledo Medical Center in Toledo, Ohio for their assistance with recruitment. All authors declare no potential conflicts of interest. This work was supported by National Institutes of Health [RO1A081558 and RO1AG045973 to M.A.J.W.]

\section{References}

1. Cohen AL, Harrison LH, Farley MM, Reingold AL, Hadler J, et al. (2010) Prevention of invasive pneumococcal disease among HIV-infected adults in the era of childhood pneumococcal immunization. AIDS 24: 2253-2262.

2. Flannery B, Heffernan RT, Harrison LH, Ray SM, Reingold AL, et al. (2006) Changes in invasive Pneumococcal disease among HIV-infected adults living in the era of childhood pneumococcal immunization. Annals of internal medicine 144: 1-9.

3. Yin Z, Rice BD, Waight P, Miller E, George R, et al. (2012) Invasive pneumococcal disease among HIV-positive individuals, 2000-2009. AIDS 26: 87-94.
4. McEllistrem MC, Mendelsohn AB, Pass MA, Elliott JA, Whitney CG, et al. (2002) Recurrent invasive pneumococcal disease in individuals with human immunodeficiency virus infection. J Infect Dis 185: 1364-1368.

5. Wolter N, Cohen C, Tempia S, Madhi SA, Venter M, et al. (2014) HIV and influenza virus infections are associated with increased blood pneumococcal load: a prospective, hospital-based observational study in South Africa, 20092011. The Journal of infectious diseases 209: 56-65.

6. Mahy M, Autenrieth CS, Stanecki K, Wynd S (2014) Increasing trends in HIV prevalence among people aged 50 years and older: evidence from estimates and survey data. AIDS 28 Suppl 4: S453-459.

7. Greene M, Justice AC, Lampiris HW, Valcour V (2013) Management of human immunodeficiency virus infection in advanced age. JAMA 309: 1397-1405.

8. Samji H, Cescon A, Hogg RS, Modur SP, Althoff KN, et al. (2013) Closing the gap: increases in life expectancy among treated HIV-positive individuals in the United States and Canada. PLoS One 8: e81355.

9. Effros RB, Fletcher CV, Gebo K, Halter JB, Hazzard WR, et al. (2008) Aging and infectious diseases: workshop on HIV infection and aging: what is known and future research directions. Clinical infectious diseases: an official publication of the Infectious Diseases Society of America 47: 542-53.

10. Deeks SG (2011) HIV infection, inflammation, immunosenescence, and aging Annu Rev Med 62: 141-155.

11. Iyer AS, Ohtola JA, Westerink MA (2015) Age-related immune response to pneumococcal polysaccharide vaccination: lessons for the clinic. Expert review of vaccines 14: 85-97.

12. De Biasi S, Pinti M, Nasi M, Gibellini L, Bertoncelli L, et al. (2011) HIV-1 Infection and the Aging of the Immune System: Facts, Similarities and Perspectives. J Exp Clin Med 3: 143-50.

13. Kaplan JE, Benson C, Holmes KK, Brooks JT, Pau A, et al. (2009) Guidelines for prevention and treatment of opportunistic infections in HIV-infected adults and adolescents: recommendations from CDC, the National Institutes of Health, and the HIV Medicine Association of the Infectious Diseases Society of America. MMWR Recomm Rep 58: 1-207.

14. Centers for Disease C, Prevention (2012) Use of 13-valent pneumococcal conjugate vaccine and 23-valent pneumococcal polysaccharide vaccine for adults with immunocompromising conditions: recommendations of the Advisory Committee on Immunization Practices (ACIP). MMWR Morbidity and mortality weekly report 61: 816-819.

15. Pedersen RH, Lohse N, Østergaard L, Søgaard OS (2011) The effectiveness of pneumococcal polysaccharide vaccination in HIV-infected adults: a systematic review. HIV Med 12: 323-333.

16. Teshale EH, Hanson D, Flannery B, Phares C, Wolfe M, et al. (2008) Effectiveness of 23-valent polysaccharide pneumococcal vaccine on pneumonia in HIV-infected adults in the United States, 1998--2003. Vaccine 26: $5830-5834$

17. Crum-Cianflone NF, Huppler Hullsiek K, Roediger M, Ganesan A, Patel S, et al. (2010) A randomized clinical trial comparing revaccination with pneumococcal conjugate vaccine to polysaccharide vaccine among HIV-infected adults. The Journal of infectious diseases 202: 1114-25

18. Feikin DR, Elie CM, Goetz MB, Lennox JL, Carlone GM, et al. (2001) Randomized trial of the quantitative and functional antibody responses to a 7 -valent pneumococcal conjugate vaccine and/or 23-valent polysaccharide vaccine among HIV-infected adults. Vaccine 20: 545-553.

19. Ho YL, Brandão AP, de Cunto Brandileone MC, Lopes MH (2013) Immunogenicity and safety of pneumococcal conjugate polysaccharide and free polysaccharide vaccines alone or combined in HIV-infected adults in Brazil. Vaccine 31: 4047-4053.

20. Lesprit P, Pédrono G, Molina JM, Goujard C, Girard PM, et al. (2007) Immunological efficacy of a prime-boost pneumococcal vaccination in HIVinfected adults. AIDS 21: 2425-2434.

21. Lu CL, Chang SY, Chuang YC, Liu WC, Su CT, et al. (2014) Revaccination with 7-valent pneumococcal conjugate vaccine elicits better serologic response than 23-valent pneumococcal polysaccharide vaccine in HIV-infected adult patients who have undergone primary vaccination with 23-valent pneumococcal polysaccharide vaccine in the era of combination antiretroviral therapy. Vaccine 32: 1031-1035. 
Citation: Ohtola JA, Saul-McBeth JL, lyer AS, Leggat DJ, Khuder SA, et al. (2016) Quantitative and Functional Antibody Responses to the 13-Valent Conjugate and/or 23-Valent Purified Polysaccharide Vaccine in Aging HIV-Infected Adults. J AIDS Clin Res 7: 556. doi:10.4172/21556113.1000556

22. Peñaranda M, Payeras A, Cambra A, Mila J, Riera M; Majorcan Pneumococcal Study Group (2010) Conjugate and polysaccharide pneumococcal vaccines do not improve initial response of the polysaccharide vaccine in HIV-infected adults. AIDS 24: 1226-1228.

23. Kroon FP, van Dissel JT, Ravensbergen E, Nibbering PH, van Furth R (2000) Enhanced antibody response to pneumococcal polysaccharide vaccine after prior immunization with conjugate pneumococcal vaccine in HIV-infected adults. Vaccine 19: 886-94.

24. Leggat DJ, Thompson RS, Khaskhely NM, Iyer AS, Westerink MA (2013) The immune response to pneumococcal polysaccharides 14 and $23 \mathrm{~F}$ among elderly individuals consists predominantly of switched memory B cells. The Journal of infectious diseases 208: 101-108.

25. Khaskhely N, Mosakowski J, Thompson RS, Khuder S, Smithson SL, et al. (2012) Phenotypic analysis of pneumococcal polysaccharide-specific B cells. J Immunol 188: 2455-2463.

26. Iyer AS, Leggat DJ, Ohtola JA, Duggan JM, Georgescu CA, et al. (2015) Response to Pneumococcal Polysaccharide Vaccination in HIV-Positive Individuals on Long Term Highly Active Antiretroviral Therapy. J AIDS Clin Res 6.

27. Leggat DJ, lyer AS, Ohtola JA, Kommoori S, Duggan JM, et al. (2015) Response to Pneumococcal Polysaccharide Vaccination in Newly Diagnosed HIV-Positive Individuals. J AIDS Clin Res 6.

28. Hart M, Steel A, Clark SA, Moyle G, Nelson M, et al. (2007) Loss of discrete memory $B$ cell subsets is associated with impaired immunization responses in HIV-1 infection and may be a risk factor for invasive pneumococcal disease. Journal of immunology 178: 8212-8220.

29. D'Orsogna LJ, Krueger RG, McKinnon EJ, French MA (2007) Circulating memory B-cell subpopulations are affected differently by HIV infection and antiretroviral therapy. AIDS 21: 1747-1752.

30. Titanji K, De Milito A, Cagigi A, Thorstensson R, Grützmeier S, et al. (2006) Loss of memory $B$ cells impairs maintenance of long-term serologic memory during HIV-1 infection. Blood 108: 1580-1587.

31. Kelley CF, Kitchen CM, Hunt PW, Rodriguez B, Hecht FM, et al. (2009) Incomplete peripheral CD4+ cell count restoration in HIV-infected patients receiving long-term antiretroviral treatment. Clinical infectious diseases : an official publication of the Infectious Diseases Society of America 48: 787-794.

32. Park S, Nahm MH (2011) Older adults have a low capacity to opsonize pneumococci due to low IgM antibody response to pneumococcal vaccinations. Infect Immun 79: 314-320.

33. Ohtola JA, Khaskhely NM, Saul-Mcbeth JL, lyer AS, Leggat DJ, et al. (2016) Alterations in serotype-specific B cell responses to the 13-valent pneumococcal conjugate vaccine in aging HIV-infected adults. Vaccine 34: 451-457.

34. Musher DM, Sampath R, Rodriguez-Barradas MC. (2011) The potential role for protein-conjugate pneumococcal vaccine in adults: what is the supporting evidence? Clinical infectious diseases: an official publication of the Infectious Diseases Society of America 52: 633-640.

35. Glesby MJ, Watson W, Brinson C, Greenberg RN, Lalezari JP, et al. (2014) Immunogenicity and Safety of 13 -Valent Pneumococcal Conjugate Vaccine in HIV-Infected Adults Previously Vaccinated With Pneumococcal Polysaccharide Vaccine. The Journal of infectious diseases.
36. Moore MR, Link-Gelles R, Schaffner W, Lynfield R, Lexau C, et al. (2015) Effect of use of 13-valent pneumococcal conjugate vaccine in children on invasive pneumococcal disease in children and adults in the USA: analysis of multisite, population-based surveillance. The Lancet Infectious diseases 15: 301-309.

37. Song JY, Moseley MA, Burton RL, Nahm MH (2013) Pneumococcal vaccine and opsonic pneumococcal antibody. J Infect Chemother 19: 412-425.

38. Westerink MA, Schroeder HW Jr, Nahm MH (2012) Immune Responses to pneumococcal vaccines in children and adults: Rationale for age-specific vaccination. Aging Dis 3: 51-67.

39. Käyhty H, Auranen K, Nohynek H, Dagan R, Mäkelä H (2006) Nasopharyngeal colonization: a target for pneumococcal vaccination. Expert Rev Vaccines 5: 651-667.

40. Davis SM, Deloria-Knoll M, Kassa HT, O'Brien KL (2013) Impact of pneumococcal conjugate vaccines on nasopharyngeal carriage and invasive disease among unvaccinated people: review of evidence on indirect effects. Vaccine 32: 133-145

41. Weil-Olivier C, Gaillat J (2014) Can the success of pneumococcal conjugate vaccines for the prevention of pneumococcal diseases in children be extrapolated to adults? Vaccine 32: 2022-2026.

42. Gordon SB, Kayhty H, Molyneux ME, Haikala R, Nurkka A, et al. (2007) Pneumococcal conjugate vaccine is immunogenic in lung fluid of HIV-infected and immunocompetent adults. J Allergy Clin Immunol 120: 208-210.

43. Nieminen T, Eskola J, Kayhty H (1998) Pneumococcal conjugate vaccination in adults: circulating antibody secreting cell response and humoral antibody responses in saliva and in serum. Vaccine 16: 630-636.

44. Breiman RF, Keller DW, Phelan MA, Sniadack DH, Stephens DS, et al (2000) Evaluation of effectiveness of the 23-valent pneumococcal capsular polysaccharide vaccine for HIV-infected patients. Archives of internal medicine 160: 2633-2638.

45. French N, Nakiyingi J, Carpenter LM, Lugada E, Watera C, et al. (2000) 23-valent pneumococcal polysaccharide vaccine in HIV-1-infected Ugandan adults: double-blind, randomised and placebo controlled trial. Lancet 355 : 2106-2111.

46. French N, Gordon SB, Mwalukomo T, White SA, Mwafulirwa G, et al. (2010) A trial of a 7-valent pneumococcal conjugate vaccine in HIV-infected adults. $\mathrm{N}$ Engl J Med 362: 812-822.

47. Klugman KP, Madhi SA, Huebner RE, Kohberger R, Mbelle N, et al. (2003) A trial of a 9-valent pneumococcal conjugate vaccine in children with and those without HIV infection. N Engl J Med 349: 1341-1348.

48. Conaty S, Watson L, Dinnes J, Waugh N (2004) The effectiveness of pneumococcal polysaccharide vaccines in adults: a systematic review of observational studies and comparison with results from randomised controlled trials. Vaccine 22: 3214-3224.

49. Vila-Corcoles A, Ochoa-Gondar O (2013) Preventing pneumococcal disease in the elderly: recent advances in vaccines and implications for clinical practice. Drugs \& aging 30: 263-276.

50. Bonten MJ, Huijts SM, Bolkenbaas M, Webber C, Patterson S, et al. (2015) Polysaccharide conjugate vaccine against pneumococcal pneumonia in adults. The New England journal of medicine 372: 1114-1125. 